QMW-95-25

hep-th/9506194

June 1995

\title{
STRING-STRING DUALITY IN TEN DIMENSIONS
}

\author{
C.M. HulL \\ Physics Department, Queen Mary and Westfield College, \\ Mile End Road, London E1 4NS, U.K.
}

\begin{abstract}
The heterotic string occurs as a soliton of the type I superstring in ten dimensions, supporting the conjecture that these two theories are equivalent. The conjecture that the type IIB string is self-dual, with the strong coupling dynamics described by a dual type IIB theory, is supported by the occurrence of the dual string as a Ramond-Ramond soliton of the weakly-coupled theory.
\end{abstract}




\section{Introduction}

Recent evidence suggests that, in many cases, string theories that appear very different perturbatively may in fact be equivalent when non-perturbative effects are taken into account [1-4]. For example, the heterotic string compactified to six dimensions on $T^{4}$ is conjectured to be equivalent to the type II string compactified on $K_{3}$ [1]. Soon after the heterotic string was discovered, it was conjectured by Green and Witten that the heterotic string with gauge group $S O(32)$ and the type I string with the same gauge group might be equivalent; indeed, they have the same low-energy effective field theory. The heterotic string spectrum contains spinor representations of $S O(32)$, but there are no such representations in the perturbative spectrum of the type I string, so such representations would have to arise as solitons of the type I theory. It was pointed out in [3] that if such a relation were to hold, then the strong coupling limit of one should correspond to the weak coupling limit of the other. This is certainly the case at the level of the low-energy effective field theories [3]. In this paper further evidence supporting this conjecture, and the conjectured self-duality of type IIB strings [1,3], will be provided.

Evidence supporting the conjectured equivalence of type II and heterotic strings in six dimensions was presented in $[5,6]$, where it was shown that the heterotic string emerges as a non-singular soliton of the type II string compactified on $K_{3}$ with the correct zero-mode structure. It is believed that the type II string should also arise as a soliton of the six-dimensional heterotic string, but the zero-mode analysis of the natural solution in this context has so far proved problematic [6]. Such situations in which the strong coupling limit of one string theory is the weak coupling limit of another 'dual' string theory were discussed earlier by Duff [7], who called the phenomenon string-string duality. In the same spirit, it will be shown here that the ten-dimensional heterotic string arises as a soliton of the type I string, which is evidence in favour of the conjectured heterotic/type I equivalence. To complete the picture, it would be desirable to obtain the type I string as a soliton of the 
heterotic string theory, but as yet no such soliton is known.

The heterotic string in four dimensions is believed to be self-dual: the strong coupling limit is described by a dual heterotic string with electric and magnetic charges interchanged, generalising to string theory the conjectured MontonenOlive self-duality of $N=4$ supersymmetric Yang-Mills. This is a consequence of the conjectured $S L(2, \mathbb{Z})$ S-duality of the theory [8]. In [1], it was conjectured that the type IIB superstring in ten dimensions also has an $S L(2, \mathbb{Z})$ duality symmetry. This would imply that this theory is also self-dual, as the $S L(2, \mathbb{Z})$ symmetry includes a transformation that interchanges weak and strong coupling regimes. However, whereas the four-dimensional heterotic string duality interchanges electric and magnetic charges, this ten-dimensional duality interchanges Neveu-Schwarz/Neveu-Schwarz (NS-NS) and Ramond-Ramond (RR) charges. It will be shown here that the weakly coupled type IIB string has a solitonic string that is interchanged with the fundamental type IIB string by duality and which corresponds to the fundamental string of the dual strongly coupled theory. This constitutes further evidence in favour of the conjectured self-duality of the type IIB string.

\section{Solitons of Heterotic and Type I Strings}

The heterotic string low-energy effective action includes the bosonic terms

$$
\int d^{10} x \sqrt{-g} e^{-2 \Phi}\left(R+4 \partial_{\mu} \Phi \partial^{\mu} \Phi-\frac{1}{4} F_{\mu \nu}^{I} F^{I \mu \nu}-\frac{1}{12} H_{\mu \nu \rho} H^{\mu \nu \rho}\right)
$$

while the corresponding action for the type I string is [3]

$$
\int d^{10} x \sqrt{-g}\left(e^{-2 \Phi}\left(R+4 \partial_{\mu} \Phi \partial^{\mu} \Phi\right)-\frac{1}{4} e^{-\Phi} F_{\mu \nu}^{I} F^{I \mu \nu}-\frac{1}{12} H_{\mu \nu \rho} H^{\mu \nu \rho}\right)
$$

with different dilaton dependence. The substitution

$$
g_{\mu \nu} \rightarrow e^{\Phi} g_{\mu \nu}, \quad \Phi \rightarrow-\Phi
$$

in the heterotic action (2.1) gives the type I action (2.2). The dilaton sign flip 
implies that the heterotic string coupling constant is inversely related to the type I string coupling constant, so that the weak coupling limit of one indeed corresponds to the strong coupling limit of the other [3].

The heterotic string has a neutral string soliton given by [9]

$$
\begin{aligned}
& d s^{2}=\left(1-\left(\frac{a}{r}\right)^{6}\right)\left(-d t^{2}+d \sigma^{2}\right)+\left(1-\left(\frac{a}{r}\right)^{6}\right)^{-5 / 3} d r^{2}+r^{2}\left(1-\left(\frac{a}{r}\right)^{6}\right)^{1 / 3} d \Omega_{7}^{2} \\
& e^{2 \Phi}=1-\left(\frac{a}{r}\right)^{6}, \quad H=6 a^{6} e^{2 \Phi} * \epsilon_{7}
\end{aligned}
$$

where $\sigma$ is the coordinate parameterising the string, * denotes the Hodge dual, $d \Omega_{7}^{2}$ is the line element on the unit seven-sphere and $\epsilon_{7}$ is its volume form. This is the extremal limit of the black string solutions of [10] and is singular on the horizon $r=a$. It also preserves half of the ten-dimensional supersymmetry and saturates the corresponding Bogomolnyi bound [9]. The solution describes the space-time outside a fundamental string source [9] and might be thought of as a soliton which should be identified with the fundamental string.

There is also a neutral string solution of the type I string action (2.2) given by

$$
\begin{aligned}
d s^{2} & =A^{1 / 2}\left(-d t^{2}+d \sigma^{2}\right)+A^{-1 / 6}\left(A^{-2} d r^{2}+r^{2} d \Omega_{7}^{2}\right) \\
e^{-2 \Phi} & =A, \quad A \equiv 1-\left(\frac{a}{r}\right)^{6}, \quad H=6 a^{6} e^{-2 \Phi} * \epsilon_{7}
\end{aligned}
$$

This is related to (2.4) by the conformal rescaling of the metric and dilaton sign flip (2.3). The metric is the special case $\alpha=-1 / 2$ of the rescaled metric

$$
\tilde{g}_{\mu \nu}=e^{2 \alpha \Phi} g_{\mu \nu}
$$

and the singularity or otherwise of this class of metrics will now be considered.

The metric

$$
d \tilde{s}^{2}=A^{\alpha+1}\left(-d t^{2}+d \sigma^{2}\right)+A^{\alpha+1 / 3}\left(A^{-2} d r^{2}+r^{2} d \Omega_{7}^{2}\right)
$$

(with $A$ given by (2.5)) has a potential singularity at $r=a$. To investigate the 
behaviour near $r=a$, it is convenient to follow [11-13] and define a new coordinate

$$
\lambda=\frac{6(r-a)}{a}
$$

so that the metric becomes

$$
d \tilde{s}^{2}=\lambda^{\alpha+1}\left(-d t^{2}+d \sigma^{2}\right)[1+O(\lambda)]+\lambda^{\alpha+1 / 3}\left(\frac{a^{2}}{36} \lambda^{-2} d \lambda^{2}+a^{2} d \Omega_{7}^{2}\right)[1+O(\lambda)]
$$

Further defining

$$
\rho=\frac{1}{6} a \ln \lambda
$$

and suppressing the $O(\lambda)$ terms, the metric tends asymptotically to

$$
d \tilde{s}^{2} \sim e^{\frac{6}{a}(\alpha+1) \rho}\left(-d t^{2}+d \sigma^{2}\right)+e^{\frac{6}{a}(\alpha+1 / 3) \rho}\left(d \rho^{2}+a^{2} d \Omega_{7}^{2}\right)
$$

as $r \rightarrow a, \rho \rightarrow-\infty$. Asymptotically, the dilaton is given by

$$
\Phi \sim \frac{3}{a} \rho
$$

so that there is a linear dilaton (in these coordinates) which blows up as $r \rightarrow a$, $\rho \rightarrow-\infty$. Thus there is a dilaton singularity as well as a potential curvature singularity at $r=a$, so that the solution can only be regarded as non-singular if $r=a$ is an infinite geodesic distance from all points with $r>a$.

There are a number of different cases, depending on the value of $\alpha$. The asymptotic metric is a warped product of two-dimensional Minkowski-space $M_{2}$ with coordinates $t, \sigma$ and a cylindrical 'throat' $C_{8}=\mathbb{R} \times S^{7}$ which has a sevensphere cross-section of radius $a$. If $\alpha<-1$ the conformal factors blow up both $M_{2}$ and $C_{8}$ as $\rho \rightarrow-\infty$, while if $-1<\alpha<-1 / 3$ the throat opens up (like the bell of a trumpet) and the $M_{2}$ shrinks. If $\alpha>-1 / 3$ both $C_{8}$ and $M_{2}$ contract, so that the throat pinches off. If $\alpha<-1 / 3$, then it follows from the asymptotic 
form of the metric (2.11) that the distance along a space-like geodesic lying entirely within $C_{8}$ from any point with finite $\rho$ to $\rho=-\infty$, which corresponds to $r=a$, is infinite. Thus a solution with $\alpha<-1 / 3$, and in particular the type I solution (2.5) with $\alpha=-1 / 2$, has non-singular spatial sections of constant $t, \sigma$. For $\alpha \geq-1 / 3$, it is a finite distance to $r=a$ along space-like geodesics, so that the solution is singular. In the special case $\alpha=-1 / 3$, the asymptotic metric is the standard metric on $S^{7} \times A d S^{3}$, where $A d S^{3}$ is three-dimensional anti-de Sitter space [12]. In this case, $\tilde{g}_{\mu \nu}$ is the so-called 5 -brane metric and the string soliton has a nonsingular geometry which interpolates between $d=10$ Minkowski space $M_{10}$ and the $S^{7} \times A d S^{3}$ string solution with linear dilaton, but has a dilaton that diverges on $r=a[12]$.

Although $r=a$ cannot be reached along space-like geodesics of finite length for $\alpha<-1 / 3$, there remains the possibility of the singularity being reached by timelike or null geodesics of finite length. In fact, the singularity can be reached by a finite-length time-like geodesic if $\alpha>-2 / 3$ [14]. For a time-like radial geodesic $r(t)$, the proper time $\tau$ is defined by

$$
d \tau^{2}=A^{\alpha+1} d t^{2}-A^{\alpha-5 / 3} d r^{2}
$$

and energy conservation implies that

$$
A^{\alpha+1} \frac{d t}{d \tau}=\epsilon
$$

for some constant $\epsilon$. This implies

$$
\left(\frac{\epsilon^{2}}{A^{\alpha+1}}-1\right) d \tau^{2}=A^{\alpha-5 / 3} d r^{2}
$$

Near $r=a, \lambda=0, A \sim \lambda$ and if $\alpha>-1$, then (2.15) implies

$$
\frac{d \tau}{d \lambda} \sim \frac{a}{6 \epsilon} \lambda^{\alpha-1 / 3}
$$

so that the proper time along the geodesic from the singularity $\lambda=0$ to some 
small value $\lambda=\Lambda$ is

$$
\tau \sim \frac{a}{6 \epsilon} \int_{0}^{\Lambda} \frac{d \lambda}{\lambda^{1 / 3-\alpha}}
$$

and this is finite if $\alpha>-2 / 3$. In particular, for the open string soliton (2.5), $\alpha=-1 / 2$ and the singularity can be reached along time-like geodesics.

The heterotic string action (2.1) also has a five-brane solution $[15,16]$

$$
\begin{aligned}
d s^{2} & =-d t^{2}+d x^{i} d x^{i}+B^{-2} d r^{2}+r^{2} d \Omega_{3}^{2} \\
e^{-2 \Phi} & =B, \quad B=1-\left(\frac{a}{r}\right)^{2}, \quad H=2 a^{2} \epsilon_{3}
\end{aligned}
$$

where $x^{i}(i=1, \ldots 5)$ are coordinates on the five-brane, $d \Omega_{3}^{2}$ is the line element on the unit three-sphere and $\epsilon_{3}$ is its volume form. This is a stable non-singular soliton that saturates a Bogomolnyi bound and interpolates between the Minkowski space $M_{10}$ and the $S^{3}$ compactification to $d=7$ Minkowski space with a linear dilaton [11]. Consider the rescaled metric (2.6), which in this case is given by

$$
d \tilde{s}^{2}=B^{-\alpha}\left(-d t^{2}+d x^{i} d x^{i}+B^{-2} d r^{2}+r^{2} d \Omega_{3}^{2}\right)
$$

and has a potential singularity at $r=a$. To investigate the behaviour near $r=a$, it is convenient to again follow [11-13] and define new coordinates

$$
\lambda=\frac{2(r-a)}{a}, \quad \rho=\frac{1}{2} a \ln \lambda
$$

so that suppressing the $O(\lambda)$ terms, the metric tends asymptotically to

$$
d \tilde{s}^{2} \sim e^{-\frac{2 \alpha}{a} \rho}\left(-d t^{2}+d x^{i} d x^{i}+d \rho^{2}+a^{2} d \Omega_{3}^{2}\right)
$$

as $r \rightarrow a, \rho \rightarrow-\infty$. Asymptotically, the dilaton is given by $\Phi \sim-\frac{1}{a} \rho$ so that there is a linear dilaton which blows up as $r \rightarrow a, \rho \rightarrow-\infty$. From the asymptotic form (2.21), the distance to $r=a$ along space-like geodesics is finite if $\alpha<0$. Thus although the five-brane is a non-singular solution of the heterotic string $(\alpha=0)$, it is singular in the open string metric $(\alpha=-1 / 2)$ and the five-brane metric $(\alpha=-1 / 3)$. 


\section{Solitons of the Type IIB String}

The massless bosonic fields of the type IIB string consist of the graviton $\bar{g}_{\mu \nu}$, the NS-NS anti-symmetric tensor gauge field $b_{\mu \nu}^{1}$, the RR one $b_{\mu \nu}^{2}$, a four-form with self-dual field strength $G_{\mu \nu \rho \sigma \tau}$ and a complex scalar $\lambda=\psi+i e^{-\Phi}$, where $\Phi$ is the dilaton. There is no action that gives the full low-energy field equations, but for

solutions with $G_{\mu \nu \rho \sigma \tau}=0$, the remaining bosonic low-energy field equations can be obtained by varying the action

$$
\int d^{10} x \sqrt{-\bar{g}}\left(\bar{R}+4 t r \partial_{\mu} \mathcal{S} \partial^{\mu} \mathcal{S}^{-1}-\frac{1}{12} \operatorname{tr}\left[\mathcal{H}_{\mu \nu \rho}^{t} \mathcal{S} \mathcal{H}^{\mu \nu \rho}\right]\right)
$$

where $\bar{g}_{\mu \nu}$ is the canonical Einstein metric and

$$
\mathcal{S}=\frac{1}{\lambda_{2}}\left(\begin{array}{cc}
|\lambda|^{2} & \lambda_{1} \\
\lambda_{1} & 1
\end{array}\right), \quad \mathcal{H}_{\mu \nu \rho}=\left(\begin{array}{c}
H_{\mu \nu \rho}^{1} \\
H_{\mu \nu \rho}^{2}
\end{array}\right), \quad \lambda=\lambda_{1}+i \lambda_{2}, \quad H^{i}=d b^{i}
$$

The type IIB supergravity field equations are invariant under $S L(2, \mathbb{R})$ which leaves $G$ and $\bar{g}_{\mu \nu}$ invariant and acts on the remaining fields by

$$
\mathcal{S} \rightarrow \Lambda \mathcal{S} \Lambda^{t}, \quad \mathcal{H} \rightarrow \Lambda^{-1} \mathcal{H}
$$

where $\Lambda$ is a $2 \times 2$ matrix in $S L(2, \mathbb{R})$. In the string metric $g_{\mu \nu}=e^{\Phi / 2} \bar{g}_{\mu \nu}$, the action is [17]

$$
\int d^{10} x \sqrt{-g}\left[e^{-2 \Phi}\left(R+4 \partial_{\mu} \Phi \partial^{\mu} \Phi-\frac{1}{12}\left(H^{1}\right)^{2}\right)-\frac{1}{2} \partial_{\mu} \psi \partial^{\mu} \psi-\frac{1}{12}\left(H^{2}+\psi H^{1}\right)^{2}\right]
$$

and the dilaton doesn't couple to the RR fields $\psi, H^{2}$ [17]. Note that there is a consistent truncation to a type I action given by setting $H^{1}=0, \psi=0$.

There are solutions to the field equations derived from (3.4) with $\psi=0$ and either $H^{1}=0$ or $H^{2}=0$. The action that gives the field equations with $\psi=0$ 
and $H^{2}=0$ is precisely the heterotic string action (2.1) with $F=0$ and $H=H^{1}$, while the one that gives the field equations with $\psi=0$ and $H^{1}=0$ is precisely the type I string action (2.2) with $F=0$ and $H=H^{2}$. This immediately implies that the type IIB theory has two string and two five-brane solutions $[1,2,4,10]$. There is a singular NS-NS string and non-singular five-brane given by (2.4), (2.18) respectively with $H=H^{1}$, and there is a RR string given by (2.5) with $H=H^{2}$ and a singular RR five-brane given by (2.19) with $H=H^{2}$ and $\alpha=-1 / 2$. The action of the $S L(2, \mathbb{Z})$ transformation given by $(2.19)$ with $\Lambda=\left(\begin{array}{cc}0 & 1 \\ -1 & 0\end{array}\right)$ interchanges $H^{1}$ and $H^{2}$ while $\Phi \rightarrow-\Phi$ (with $\psi=0$ ) so that the two string solutions and the two five-brane solutions are interchanged.

\section{Charged Strings, Zero Modes and String-String Duality}

Before considering the zero modes of the ten-dimensional solutions, it will be useful to first discuss the six-dimensional solutions $[5,6]$. The toroidally compactified six-dimensional heterotic string has an abelian $U(1)^{24}$ gauge symmetry at generic points in the $O(4,20) / O(4) \times O(20)$ moduli space. The fundamental string solution [9] has two Killing vectors acting without fixed points (generating translations in time $t$ and along the string) so that there is an $O(6,22)$ solution generating group: acting on the solution with this group generates new solutions. A subgroup $O(4,1) \times O(1,20)$ preserves the boundary conditions and an $O(4) \times O(20)$ subgroup of this acts trivially [18]. Acting with transformations in the 24-dimensional coset $O(4,1) / O(4) \times O(1,20) / O(20)$ generates the charged strings solutions of $[5,19]$ which carry $U(1)^{24}$ electric charge. They also carry an electric current proportional to the charge per unit length, so that these strings are accompanied by both electric and magnetic fields. These solutions are parameterised by the 24 electric charge densities, and there are 24 collective coordinates conjugate to these charges. These coordinates are periodic, since the charges are quantised, and they are represented by 24 world-sheet bosonic coordinates, which are chiral (right-moving) because the charge and current densities are equal [19]. In addition, there are the expected four 
bosonic zero modes corresponding to translations in the four dimensions transverse to the string, and four fermionic ones corresponding to the unbroken supersymmetries; these are represented by left-moving world-sheet spinors. The type II theory compactified on $K_{3} \times T^{2}$ has a string soliton with the same zero mode structure $[5,6]$, which justifies identifying it with the heterotic string. In particular, the neutral string soliton has charged string generalisations parameterised by the 24 electric charge densities, which equal the current densities, so that the corresponding world-sheet theory has 24 chiral bosons [5]. The type II origin of these zero modes was discussed in [6].

The situation is different at the special points in the $O(4,20) / O(4) \times O(20)$ moduli space at which the gauge symmetry is enhanced to $\hat{G}=U(1)^{4} \times G$ where $G$ is a rank 20 non-abelian group. The low-energy effective field theory has a non-abelian gauge symmetry, but there are solutions in which only gauge fields taking values in a 24-dimensional Cartan subalgebra are non-trivial. The effective theory for such solutions is the abelian theory considered above, so that there is a 24 parameter space of charged string solutions. As there is such a space for every Cartan subalgebra, there is a charged string carrying each of the $d_{G} \equiv \operatorname{dim}(\hat{G})$ charges. There should be a chiral bosonic zero mode conjugate to each of these charges, but only 24 of them are expected to constitute a mutually commuting set. Recall that the chiral sector of the heterotic string can be formulated using the right-moving sector of a Wess-Zumino-Witten (WZW) model (of level one) instead of the usual chiral bosons or fermions [20], so that here the natural structure to expect for the world-sheet theory describing the extra heterotic zero modes is a chiral $\hat{G}$ WZW model (in addition to the usual bosonic translation zero-modes and the fermionic modes corresponding to unbroken supersymmetry). The extra $d_{G}-24$ zero modes can also be understood in terms of the type II string. At the special points in moduli space certain solitonic states become massless [3,4], and the soliton has zero-modes in which these extra massless fields are excited. The solitonic states that become massless are six-dimensional black holes resulting from $p$-brane solitons of the ten-dimensional theory wrapped around $K_{3}$ homology 
cycles, and they become massless at points in moduli space at which the homology cycles collapse to zero area $[3,4]$.

The fundamental heterotic string solution of the $S O(32)$ or $E_{8} \times E_{8}$ heterotic string and the heterotic soliton of the $S O(32)$ type I string have a zero mode structure similar to that of the six-dimensional heterotic string at enhanced symmetry points. Solutions of the effective $N=1$ supergravity theory coupled to 16 abelian vector multiplets are solutions of the full theory in which only 16 of the 496 gauge fields, taking values in a Cartan subalgebra, are non-zero. For any choice of Cartan subalgebra, acting on the fundamental heterotic string solution (2.4) with the solution generating transformations in the coset $O(16,1) / O(16)$ gives charged string solutions [19] parameterised by 16 electric charges. The collective coordinates corresponding to these 16 charges are 16 periodic chiral bosons. Repeating this with different choices of Cartan subalgebra, one obtains charged strings carrying any of the 496 types of charge, and the corresponding chiral bosonic zero-modes should be described by a level one WZW model with group $S O(32)$ or $E_{8} \times E_{8}$. These, together with the eight bosonic zero modes corresponding to transverse translations and the eight fermionic zero modes corresponding to the unbroken supersymmetries give a zero-mode structure described by the Green-Schwarz light-cone heterotic string with the heterotic degrees of freedom described by a chiral WZW model, as in [20].

Acting on this 496-dimensional space of charged string generalisations of (2.4) with the field transformation (2.3) (for gauge group $S O(32)$ ) gives a 496dimensional space of charged string solutions of the type I string action (2.2) generalising the solution (2.5). These solutions have the same singularity structure as (2.5) and have equal charge and current densities. The zero mode structure is that of the $S O(32)$ heterotic string: it is described by a light-cone string with 8 bosons, 8 fermions and an $S O(32)$ WZW model, justifying the identification of this soliton with the heterotic string.

It remains an open question as to whether singular solutions can be acceptable 
as solitons and, if so, what types of singularity can be allowed. The form of a solution of an effective theory can only be trusted down to length scales corresponding to the masses of the lightest fields that have been integrated out, and including such massive fields can drastically change the short-distance structure of a solution and in some cases remove the singularity. Thus even if a solution is singular, the singularity might be removed by including extra fields in the effective theory. In six dimensions, the solitons considered in $[5,6]$ are non-singular. However, in ten dimensions the solution (2.5) has non-singular space-like sections, but has a singularity at $r=a$ that can be reached along a finite time-like geodesic, so that the question arises as to whether it should be regarded as a soliton. It was argued in [4] that, in the case of the type IIB string, both strings and both five-branes should be included in the Bogomolnyi spectrum, despite the singularity of some of them as solutions of the weakly coupled effective theory. Since the uncharged sector of the type I theory is contained within the type IIB theory, a similar argument implies that the string and five-brane should both be included in the Bogomolnyi spectrum of the type I string. More specifically, the arguments of [4] imply that the weakly coupled type I or IIB string should have a string-like Bogomolnyi state carrying the same charges as (2.5) and so which should be approximately described by the solution (2.5) at large distances, and have the same zero-mode structure. However, at short distances, and in particular near $r=a$, the identification of the Bogomolnyi state with the classical solution (2.5) is no longer trustworthy, and other effects might be expected to come into play.

The singular solution (2.4) of the heterotic string or type IIB string is the solution generated by an elementary string source, and so should not be regarded as giving rise to any new states. The string soliton (2.5) of the type I string has the zero mode structure of a heterotic string and in the strong coupling limit becomes an elementary excitation of the dual theory, which is the heterotic string theory. Similarly, the RR string soliton of type IIB is an elementary excitation of the dual type IIB theory that emerges at strong coupling, while the NS-NS string becomes a soliton of the dual theory. Indeed, the $S L(2, \mathbb{Z})$ duality implies that the zero- 
mode structure of the RR string soliton should be exactly that required for it to be identified with the fundamental string of the strongly coupled dual theory. It is interesting that in these theories, the strongly coupled dual theory appears to be

obtained by a string/string duality rather than a string/five-brane duality of the type proposed in [21].

Note Added: After this work was completed, a related paper appeared [22] discussing similar issues.

Acknowledgements: I would like to thank Michael Green, Ashoke Sen and Paul Townsend for valuable discussions.

\section{REFERENCES}

1. C.M. Hull and P.K. Townsend, Nucl. Phys. B438 (1995) 109.

2. P.K. Townsend, Phys. Lett. B350, (1995) 184.

3. E. Witten, String Theory Dynamics in Various Dimensions, hep-th/9503124.

4. C.M. Hull and P.K. Townsend, Enhanced Gauge Symmetries in Superstring Theories (revised), hep-th/9505073.

5. A. Sen, String-String Duality Conjecture in Six Dimensions and Charged Strings, hep-th/9504027.

6. J.A. Harvey and A. Strominger, The Heterotic String is a Soliton, hepth/9504047.

7. M.J. Duff, Strong/Weak Coupling Duality from the Dual String, hepth/9501030.

8. A. Font, L. Ibanez, D. Lust and F. Quevedo, Phys. Lett. B249 (1990) 35; S.J. Rey, Phys. Rev. D43 (1991) 526; J.H. Schwarz and A. Sen, Nucl. Phys. B411 (1994) 35; Phys. Lett. 312B (1993) 105; A. Sen, Int. J. Mod. Phys. A9 (1994) 3707. 
9. A. Dabholkar, G.W. Gibbons, J.A. Harvey and F. Ruiz-Ruiz, Nucl. Phys. B340 (1990) 33.

10. G.T. Horowitz and A. Strominger, Nucl. Phys. B360 (1991) 197.

11. G.W. Gibbons and P.K. Townsend, Phys. Rev. Lett. 7 (1993) 3754.

12. M.J. Duff, G.W. Gibbons and P.K. Townsend, Phys. Lett. 332 B (1994) 321.

13. G.W. Gibbons, G.T. Horowitz and P.K. Townsend, Class. Quantum Grav. $12(1995) 297$.

14. P.K. Townsend, private communication.

15. C. Callan, J. Harvey and A. Strominger, Nucl. Phys. B359 (1991) 611.

16. M.J. Duff and J.X. Lu, Nucl. Phys. B354 (1991) 141; Phys. Lett. 273B (1991) 409.

17. E. Bergshoeff, C.M.Hull and T. Ortin, Duality in the Type II Superstring Effective Action, hep-th/9504081.

18. H. Hassan and A. Sen, Nucl. Phys. B375 (1992) 103, hep-th/9109038; H. Hassan, hep-th/9408060

19. A. Sen, Nucl. Phys. B388 (1992) 457.

20. S.J. Gates and W. Siegel, Phys. Lett. 206B (1988) 631; S.J. Gates, S.V. Ketov, S.M. Kuzenko and O.A. Soloviev, Nucl. Phys. B362 (1991) 199.

21. A. Strominger, Nucl. Phys. B343 (1990) 167.

22. A. Dabholkar, Ten-Dimensional Heterotic String as a Soliton, hepth/9506160. 